\title{
Effect of postprandial modulation of glucose availability: short- and long-term analysis
}

\author{
Julie-Anne Nazare ${ }^{1,2,3,4,5,6}$, Alexis de Rougemont ${ }^{1,2,3,4,5,6}$, Sylvie Normand ${ }^{1,2,3,4,5,6}$, Valérie Sauvinet ${ }^{1,2,3,4,5,6}$, \\ Monique Sothier ${ }^{1,2,3,4,5,6}$, Sophie Vinoy ${ }^{7}$, Michel Désage ${ }^{1,2,3,4,5,6}$ and Martine Laville 1,2,3,4,5,6* $^{\text {. }}$ \\ ${ }^{1}$ Centre de Recherche en Nutrition Humaine de Rhône-Alpes (CRNHRA), Pavillon médical, Centre hospitalier Lyon Sud, \\ 165 chemin du grand Revoyet, 69495 Pierre-Bénite, France \\ ${ }^{2}$ Université de Lyon, 69622 Lyon, France \\ ${ }^{3}$ INSA de Lyon, RMND, 69621 Villeurbanne, France \\ ${ }^{4}$ INSERM U870, 69921 Oullins, France \\ ${ }^{5}$ INRA U1235, 69921 Oullins, France \\ ${ }^{6}$ Hospices Civils de Lyon, 69437 Lyon, France \\ ${ }^{7}$ Danone Vitapole, Paris, France \\ (Received 10 June 2009 - Revised 27 October 2009 - Accepted 11 November 2009 - First published online 24 December 2009)
}

\begin{abstract}
Low glycaemic index (LGI) foods have been proposed as potential means to decrease postprandial glucose excursions and thus to improve diabetes management. We modulated glucose availability of cereal products and thus their glycaemic index to study the metabolic effect of LGI foods on daylong glucose control acutely and in the long term following a 5-week GI intervention diet in free-living subjects. In this randomised, parallel trial, two groups of nineteen overweight subjects followed an ad libitum 5-week intervention diet in which usual starch was replaced by either LGI or high GI (HGI) starch. During the exploration days (days 1 and 36), subjects ate their assigned ${ }^{13} \mathrm{C}$-labelled test breakfast (LGI or HGI), and total and exogenous glucose kinetics (using stable isotopes), postprandial concentrations of glucose, insulin, lipid profile and nutrient oxidation were assessed after the test breakfast and a standardised lunch. At day 1, LGI breakfast significantly decreased post-breakfast glycaemic response with a parallel decrease in exogenous and total glucose appearance $(P<0.05)$. Post-lunch and post-breakfast glycaemic responses were positively correlated $(r 0.79, P<0 \cdot 0001)$. Following the 5-week diet, difference between the groups in terms of glucose kinetics and response was maintained (no significant interaction group $\times$ time) but tended to decrease over time for the post-breakfast glycaemic response. Post-lunch and post-breakfast glycaemic responses remained positively correlated $(r 0.47, P=0.004)$. Modulation of postprandial glucose availability at breakfast decreased plasma exogenous glucose appearance and improved glucose control at the subsequent lunch. After 5 weeks, these effects were maintained in healthy subjects but remained to be confirmed in the longer term.
\end{abstract}

Glucose availability: Glucose kinetics: Stable isotope analysis: Second-meal effect: Short- and long-term analysis

Rising postprandial glucose excursions are associated with greater risk of developing metabolic disorders, CVD and diabetes $^{(1)}$. There has been a growing interest over recent years in the relation between the consumption of low glycaemic index (GI) or reduced glycaemic load (GL) products and their effect on the management of postprandial glycaemic profile as they have been proposed as potential tools to improve management of diabetes mellitus or to decrease risks of heart disease ${ }^{(2-4)}$. The intake of low GI (LGI) diets is associated with improved glycaemic control, more particularly among subjects with the poorest glycaemic control ${ }^{(5)}$. Several studies have shown that low GI diets could enhance glucose tolerance with a corresponding improvement in insulin sensitivity. More particularly, low GI meals have been shown to improve acute postprandial glucose tolerance and reduce insulin response, also at a subsequent meal (the 'second-meal effect' $)^{(6-10)}$. But, evidence is missing as to conclude that the acute reduction in blood glucose response elicited by low GI foods may persist in the long term, as already reported ${ }^{(11)}$. Some studies showed differences in HbA1c after LGI intervention ${ }^{(12)}$, whereas some other studies did not show any effect on $\mathrm{HbA1c}$ in type 2 diabetic subjects with optimal glycaemic control ${ }^{(13)}$. According to several authors $^{(14,15)}$, the lower glycaemic response effect produced by low GI products could be due to a slower rate of appearance of glucose in the systemic circulation. A classification has been proposed to characterise the different carbohydrate fractions by separating them into rapidly available glucose

Abbreviations: AUC, area under the curve; EGP, endogenous glucose production; GI, glycaemic index; GL, glycaemic load; HGI, high GI; iAUC, incremental AUC;

LGI, low GI; SAG, slowly available glucose; RaE, rate of appearance of exogenous glucose; RaT, total rate of glucose appearance.

* Corresponding author: Professor Martine Laville, fax +33 4788644 62, email martine.laville@chu-lyon.fr 
and slowly available glucose (SAG), reflecting the rate at which glucose becomes available for absorption in the small intestine $^{(16)}$. A high correlation has been shown between the GI of a product and the percentage of rapidly available glucose in this product measured in vitro ${ }^{(16)}$. Such SAG-rich products could be considered as lente carbohydrates and thus are candidate tools to regulate daylong glycaemic and insulinaemic profile. The modulation of postprandial metabolic profile and more particularly of insulin response could consequently modify insulin action on fuel partitioning, glucose uptake and carbohydrate and lipid oxidations. Few intervention studies have been undertaken on the effects of dietary GI, GL or SAG on these metabolic parameters in healthy overweight adults, despite the higher relative risk of insulin resistance and type 2 diabetes in this population ${ }^{(17)}$.

Therefore, we investigated the short- and long-term (5 weeks) effects of an ad libitum low GI (LGI) or HGI diet on glucose metabolism and nutrient utilisation in overweight subjects. We determined the metabolic effect of GI and its evolution over time (weeks) by thorough analysis of glucose kinetics, insulin and lipid profiles and nutrient oxidation in response to breakfast and subsequent lunch. Preliminary results of the effect of these diets on anthropometric parameters and lipid profile have been previously published showing that the 5-week LGI diet improved weight control and cholesterol profile ${ }^{(18)}$. Specific cereal processing techniques have been set up in order to produce slowly and rapidly available starchy products with exactly the same nutrient composition but differing in GI only. The follow-up of postprandial glucose kinetics (exogenous and endogenous) was performed using glucose-stable isotope analysis, after the ingestion of a ${ }^{13} \mathrm{C}$-labelled LGI (SAG-rich) or HGI (rapidly available glucose-rich) breakfast before and after the GI dietary intervention. The metabolic adaptation to a 5-week GI dietary intervention was studied through the postprandial metabolic response to a HGI or a LGI breakfast and to a subsequent lunch in order to detect a potential 'second-meal' effect.

\section{Subjects and methods}

\section{Experimental design}

This was a parallel, randomised group trial. The two groups of non-diabetic overweight subjects followed an ad libitum 5-week dietary intervention in which they were asked to replace all starches in their routine diet by either LGI or HGI starchy foods. Both types of diets were randomly allocated according to the CONSORT guidelines.

One week before the two test days (day 1 and day 36), the subjects were asked to avoid nutrients known to be enriched in ${ }^{13} \mathrm{C}$ (maize starch and oil, cane sugar, tropical fruits and canned foods). Twenty-four hours before D1 and D36, the subjects were asked to limit physical activity, drink no alcohol-containing beverage and eat a normal evening meal.

Subjects came to the Centre de Recherche en Nutrition Humaine de Rhône-Alpes, at Hôpital Edouard Herriot on the test day at 06.30 hours following a 12 -h overnight fast.

On D1 and D36, body weight was measured with a graded scale $\left(\mathrm{SECA}^{\circledR}{ }^{\circledR}\right.$, Valenciennes, France). Intravenous cannulas were inserted into deep forearm veins in both arms for tracer infusion on one side and blood sampling on the other side. A primed, continuous infusion of $\mathrm{D}-\left[6,6-{ }^{2} \mathrm{H}_{2}\right]$ glucose $(0.0570 \mathrm{mg} / \mathrm{kg}$ per $\mathrm{min})$ was started $120 \mathrm{~min}$ before breakfast was eaten and was maintained for the next 270 min to determine the total rate of glucose appearance (RaT). The priming dose was eighty times the infusion rate over $1 \mathrm{~min}$. At time 0 , subjects ate the test breakfast (either LGI or HGI breakfast, composition in Table 1) in $15 \mathrm{~min}$. Blood samples were taken at baseline and sequentially every $15 \mathrm{~min}$ until $90 \mathrm{~min}$, then every $30 \mathrm{~min}$ until $270 \mathrm{~min}$ following ingestion of the meal and were used to determine glucose, TAG, NEFA, insulin and C-peptide concentrations and deuterium and ${ }^{13} \mathrm{C}$ glucose isotopic enrichments. At $270 \mathrm{~min}$, a standardised HGI lunch was served to the subjects and ingested in 30 min (composition in Table 1). Blood samples were taken sequentially every $30 \mathrm{~min}$ until $480 \mathrm{~min}$ and were used to

Table 1. Macronutrient composition of the high glycaemic index (HGI) breakfast, of the low glycaemic index (LGI) breakfast and of the standardised high glycaemic lunch

\begin{tabular}{|c|c|c|c|c|c|c|c|c|}
\hline & Serving size $(\mathrm{g})$ & Proteins $(\mathrm{g})$ & Lipids (g) & Total $\mathrm{CHO}(\mathrm{g})$ & SAG (\%) & RAG (\%) & Fibre & Energy (kJ) \\
\hline \multicolumn{9}{|l|}{ LGI breakfast (GI 47) } \\
\hline Half-skimmed milk & 180 & 5.8 & 2.9 & $8 \cdot 1$ & & & & 1468.58 \\
\hline Biscuits (Gl 45) & 80 & $7 \cdot 0$ & $9 \cdot 8$ & 58.6 & 26 & 40 & 1.6 & 343.09 \\
\hline Total for LGI breakfast & 260 & $12 \cdot 8$ & $12 \cdot 7$ & $66 \cdot 7$ & 26 & 40 & 1.6 & $1807 \cdot 00$ \\
\hline Energy distribution & & $12 \%$ & $26 \%$ & $62 \%$ & & & & \\
\hline \multicolumn{9}{|l|}{ HGI breakfast (GI 66) } \\
\hline Half-skimmed milk & 180 & $5 \cdot 8$ & 2.9 & $8 \cdot 1$ & & & & 1456.03 \\
\hline Flakes (GI 70) & 80 & 6.9 & $9 \cdot 5$ & $58 \cdot 6$ & $<1$ & 66 & 1.6 & 343.09 \\
\hline Total for HGI breakfast & 260 & $12 \cdot 7$ & $12 \cdot 4$ & $66 \cdot 7$ & $<1$ & 66 & 1.6 & 1794.94 \\
\hline Energy distribution & & $12 \%$ & $26 \%$ & $62 \%$ & & & & \\
\hline \multicolumn{9}{|l|}{ Standardised HGI lunch (GI 71) } \\
\hline Minced beef ( $15 \%$ fat) & 150 & $27 \cdot 0$ & $22 \cdot 0$ & $27 \cdot 4$ & & & & \\
\hline Instantaneous mashed potatoes & 200 & 4.4 & 3.4 & $30 \cdot 3$ & & & & \\
\hline White bread & 55 & 4.4 & 0.6 & 0.0 & & & & \\
\hline Camembert cheese & 30 & 6.4 & $6 \cdot 6$ & 22.5 & & & & \\
\hline Whole milk yoghurt with fruits & 125 & 4.4 & 3.4 & & & & & \\
\hline Total for HGl lunch & 560 & $46 \cdot 6$ & $36 \cdot 0$ & $80 \cdot 2$ & - & - & 5 & 3476.90 \\
\hline Energy distribution & & $22 \%$ & $39 \%$ & $39 \%$ & & & & \\
\hline
\end{tabular}


determine glucose, TAG, NEFA and insulin and C-peptide concentrations. Blood was collected in tubes maintained at $4^{\circ} \mathrm{C}$ and immediately centrifuged. Plasma was then stored at $-20^{\circ} \mathrm{C}$ until assay.

$\mathrm{V}_{\mathrm{O} 2}$ and $\mathrm{CO}_{2}$ production were monitored by indirect calorimetry (Datex Instruments, Helsinki, Finland) after breakfast ingestion (T15-T270 min) and after lunch ingestion (T300T480 min) in order to calculate total carbohydrate, lipid oxidation and respiratory exchange rate.

Subjects remained in the supine position for the duration of the study except when required to void urine (urinary nitrogen measured three times: at baseline (T120 min); post breakfast (T270 min); post lunch (T480 min)).

\section{Subjects}

As described previously, a power calculation based on change in body weight $(1 \mathrm{~kg}, \mathrm{SD}=1 \mathrm{~kg})$ as primary study endpoint was done; seventeen subjects per group provided $>80 \%$ power to detect a significant difference in weight loss between groups at the $P<0.05$ level $^{(18)}$. The pre-inclusion tests were performed on sixty-eight volunteers who responded to the recruitment campaign. Each subject underwent a screening inclusion test including measurements of body weight, height, blood pressure, waist and hip circumference; an interview regarding general health; blood sample collections (blood differential count, glycaemia, transaminases, $\gamma \mathrm{GT}$, total cholesterol, HDL and LDL cholesterols and TAG). Eating habits were also explored through dietary surveys, including consultation and advice by a trained dietitian, and dietary records. The inclusion criteria for the study were men and women aged 20-60 years, BMI $25-30 \mathrm{~kg} / \mathrm{m}^{2}$, stable body weight over the previous 3 months, normal results for pre-inclusion biological tests, sedentary or moderate physical activity and routine breakfast representing $10-25 \%$ of the daily energetic intake. Thirty-eight healthy overweight subjects (nineteen men and nineteen pre-menopausal women), aged 38.3 (SD 9.2) years, weighing 77.3 (SD 9.1) kg and with a BMI 27.3 (SD 1.5$) \mathrm{kg} / \mathrm{m}^{2}$ (means and standard deviations) were recruited for the study.

The subjects were randomised into two groups as follows: nineteen subjects in the LGI diet group (nine men and ten women) and nineteen subjects in the HGI diet group (eleven men and eight women).

All the subjects received written and oral information about the protocol and signed an informed consent document. The study was approved by the Scientific Ethics Committee of Lyon (CCPPRB Lyon A) and was in accordance with both the French 'Huriet-Serusclat' law and the Second Declaration of Helsinki. The characteristics of the thirty-eight subjects are presented in Table 2. There were no significant differences between groups at baseline.

\section{Experimental diets}

The detailed composition of the 5-week dietary intervention, the test foods and breakfasts was described previously ${ }^{(18)}$. Briefly, foods were considered as having a low GI whenever GI $<50 \%$ and a high GI whenever GI $>70 \%$. In the present study, the subjects received individual guidance by a trained clinical dietitian in order to implement their intervention
Table 2. Baseline characteristics of the subjects of the low glycaemic index (LGI) diet group and of the high glycaemic index (HGl) diet group (Mean values with their standard errors)

\begin{tabular}{|c|c|c|c|c|}
\hline & \multicolumn{2}{|c|}{$\begin{array}{l}\text { LGI diet group } \\
\qquad(n 19)\end{array}$} & \multicolumn{2}{|c|}{$\begin{array}{l}\text { HGI diet group } \\
\quad(n 19)\end{array}$} \\
\hline & Mean & SEM & Mean & SEM \\
\hline Age (years) & $36 \cdot 3$ & 2 & $40 \cdot 4$ & $2 \cdot 2$ \\
\hline Weight $(\mathrm{kg})$ & $77 \cdot 2$ & $2 \cdot 2$ & $77 \cdot 3$ & $2 \cdot 1$ \\
\hline BMI $\left(\mathrm{kg} / \mathrm{m}^{2}\right)$ & 27.5 & 0.3 & $27 \cdot 2$ & 0.3 \\
\hline Fasting glucose $(\mathrm{mmol} / \mathrm{l})$ & $5 \cdot 15$ & 0.15 & 5.31 & 0.08 \\
\hline Fasting insulin $(\mathrm{pmol} / \mathrm{l})$ & 67 & 9.5 & 61.4 & $5 \cdot 7$ \\
\hline C-peptide (nmol//) & 0.67 & 0.06 & 0.67 & 0.05 \\
\hline Total cholesterol $(\mathrm{mmol} / \mathrm{l})$ & $5 \cdot 21$ & 0.23 & $5 \cdot 2$ & $0 \cdot 18$ \\
\hline
\end{tabular}

diet, and compliance to diets was strictly controlled by dietary questionnaires. Part of the starchy products was supplied by the CRNHRA and Danone Vitapole ${ }^{\odot}$. The food provided consisted in cereal breakfast products (extruded cereals for the HGI group and plain biscuits for the LGI group), and in black bread (Harris ${ }^{\odot}$ pumpernickel) for the LGI group. According to their diet group, a detailed list was given to the subjects indicating the starches they were allowed to eat and the prohibited ones (Table 3). Subjects were asked to continue eating the same amount of starch as usual. They were also asked not to modify their dietary habits regarding the amount of food eaten, food patterns and the amount of fruits and vegetables eaten. The subjects were instructed to measure and record the exact amount eaten each day by using a 5-day food diary during the pre-inclusion period (D7-D11) and on weeks 3 (D16-D20) and 5 (D31- D35) of the study. Ingested quantities were evaluated by the dietitian using a SU.VI.MAX ${ }^{\odot}$ dietary photographic support. The macronutrient content of the test foods and 5-day dietary records was calculated using a computerised food database that included specific product-ingredient lists and recipes for test foods (latest release of $\mathrm{GENI}^{\circledR}$ software by MICRO $6^{\circledR}$ using CIQUAL ${ }^{\circledR}$ table). For the mean GI calculation of all meals from food diaries, GI data are based on the international table of GI and GL values ${ }^{(19)}$.

Table 3. Allowed starch lists according to diet group

\begin{tabular}{|c|c|}
\hline \multicolumn{2}{|c|}{ Allowed starch } \\
\hline LGI group & HGI group \\
\hline Breakfast biscuits (Gl 45) & Breakfast extruded cereals (GI 70) \\
\hline $\begin{array}{l}\text { Black bread (Harris } \\
\text { pumpernickel, Gl 50) }\end{array}$ & White bread, whole meal bread (GI 95) \\
\hline Spaghetti al dente (GI 32) & $\begin{array}{l}\text { Mashed potatoes (GI 75), peeled, } \\
\text { baked or 35-minute boiled potatoes }\end{array}$ \\
\hline Other pastas (GI 48) & (GI 70) \\
\hline $\begin{array}{l}\text { Durum wheat precooked in } \\
\text { pouch (Ebly, Gl 40) }\end{array}$ & French fries, chips (GI 75) \\
\hline Bulgur (Gl 48) & Gnocchi (GI 70) \\
\hline Semolina (GI 55) & $\begin{array}{l}\text { Flour contents, pizzas, quiche, etc. } \\
\text { (GI } 70-80 \text { ) } \\
\text { tarch (both groups) }\end{array}$ \\
\hline
\end{tabular}

Rice (GI unpredictable). Starches belonging to the other diet group list. 


\section{Breakfasts}

The breakfast products provided during the intervention trial consisted of plain biscuits (LGI) or flakes (HGI). The LGI breakfast consisted of standard biscuits $(80 \mathrm{~g})$, semi-skimmed milk $(180 \mathrm{ml})$ and non-energetic hot beverage $(\max 300 \mathrm{ml})$. The HGI breakfast consisted of flakes $(80 \mathrm{~g})$, semi-skimmed milk $(180 \mathrm{ml})$ and non-energetic hot beverage (max $300 \mathrm{ml})$. The composition of the two test meals is given in Table 1. Biscuits and extruded cereals contained different quantities of SAG, 26 and $0.4 \%$, respectively. The two breakfasts represented about $20 \%$ of daily energy intake. They were isoenergetic (about 1803.30 kJ) and contained the same amount of proteins $(12 \%)$, lipids $(26 \%)$ and carbohydrates $(62 \%)$. The only variable parameter was the GI (45 and $70 \%$ for LGI and HGI breakfasts, respectively). Both cereal products were processed by Danone (Danone Vitapole, Paris, France).

Both cereal products (biscuits and flakes) ingested on the test days (D1 and D36) were exactly the same composition as those ingested during the trial, but they were uniformly labelled with stable isotope ${ }^{13} \mathrm{C}$. For this purpose, they were manufactured with starch coming from a preparation of durum wheat semolina cultivated in a ${ }^{13} \mathrm{CO}_{2}$-enriched atmosphere, mixed with naturally rich ${ }^{13} \mathrm{C}$ sugarcane. ${ }^{13} \mathrm{C}$ enrichment of starch was adjusted to ${ }^{13} \mathrm{C}$ sugarcane enrichment.

D- $\left[6,6-{ }^{2} \mathrm{H}_{2}\right]$ glucose $(99 \mathrm{~mol} \%$ excess) was obtained from Eurisotop (Gif-sur-Yvette, France); chemical and isotopic purity was confirmed by selected-ion-monitoring GC MS analysis. It was dissolved in sterile isotonic saline $(0.9 \% \mathrm{NaCl})$ and passed through a $0 \cdot 22-\mu \mathrm{m}$ millipore filter (Millipore Corp., Bedford, MA, USA) before infusion. The preparation was pyrogen free. The concentration of deuterated glucose in the infusate was determined at the end of each test.

\section{Analytical procedures}

Metabolites and hormones. Blood samples were collected in tubes containing lithium heparinate and then centrifuged at $3645 \mathrm{~g}$ and $4^{\circ} \mathrm{C}$ for $10 \mathrm{~min}$, and the plasma was stored at $-20^{\circ} \mathrm{C}$ until analysis. Glucose, TAG and NEFA concentrations were measured with an enzymatic colorimetric method on a Cary $50 \mathrm{Bio}^{(}{ }^{\circ}$ spectrophotometer $\left(\right.$ Varian ${ }^{(}$) using a BioMérieux ${ }^{\circledR}$ Glucose RTU kit (Marcy l'Etoile, France), a BioMérieux ${ }^{\circledR}$ TG PAP 150 kit and a Wako chemicals $^{\circledR}$ NEFA-C kit (Neuss, Germany), respectively. Plasma insulin and C-peptide concentrations were determined by RIA kit (INS-IRMA Biosource, Nivelles, Belgium; Immunotech, Marseille, France, respectively).

Indirect calorimetry. Respiratory exchange measurements were integrated after breakfast ingestion and the non-protein $\mathrm{RQ}$ was calculated from $\mathrm{VO}_{2}, \mathrm{VCO}_{2}$ and measured urinary nitrogen excretion determined by chemiluminescence ${ }^{(20)}$.

Total carbohydrate and lipid oxidation were calculated according to the equation developed by Ferrannini ${ }^{(21)}$.

Isotope analysis. Plasma glucose isotopic enrichments were determined on neutral fractions of deproteinised plasma samples partially purified over sequential anioncation exchange resins, as previously described ${ }^{(22)}$. Plasma $\left[6,6-{ }^{2} \mathrm{H}_{2}\right]$ glucose was measured by organic GC-MS (Hewlett Packard 5971, Evry, France) on acetyl-bis-butane-boronyl glucose derivative using an electron impact mode and a selective monitoring of $\mathrm{m} / \mathrm{z}, 297$ and $299^{(23)}$. Plasma ${ }^{13} \mathrm{C}$ glucose enrichment was measured by GC-combustion-isotope ratio MS (GC-C-IRMS, Isoprime, GV instruments, Lyon, France) after derivatisation to pentacetyl glucose, as previously described $^{(24)}$

The ${ }^{13} \mathrm{C}$ enrichment of ingested starch (biscuits and cereals) was determined after enzymatic hydrolysis using the Thivend method $^{(25)}$, and the glucose obtained was purified by sequential anion-cation exchange chromatography before derivatisation as glucose pentacetate and analysed as previously described ${ }^{(26)}$. The ${ }^{13} \mathrm{C}$ enrichment of the derivatised glucose molecule was -34.59 (SD 0.65$) \quad \delta^{13} \mathrm{C} \%$ (1.07320 (SD $0.00071)$ atom $\%^{13} \mathrm{C}$ ) and $-35.72(\mathrm{SD} \quad 0.42) \quad \delta^{13} \mathrm{C} \%$ $\left(1.07196(\mathrm{SD} 0.00046)\right.$ atom $\left.\%{ }^{13} \mathrm{C}\right)$ for biscuits and cereals, respectively.

\section{Calculations}

Mean GI of all meals taken in a day was determined using the following equation:

$$
\mathrm{GI}_{\text {mean }}=\sum\left(\left(\mathrm{C}_{\text {food }} / \mathrm{C}_{\text {total }}\right) \times \mathrm{GI}_{\text {food }}\right),
$$

where $\mathrm{C}_{\text {food }}$ is the amount of carbohydrate (in grams) contained in each ingested food and $\mathrm{C}_{\text {total }}$ the total amount of total carbohydrate (in grams) ingested during the day. Mean GI targets were defined as $<50$ for the LGI group and $>70$ for the HGI group. GL were also determined by multiplying the total amount of total carbohydrate (in grams) by the mean GI for each food and adjusted for energy intake:

$$
\mathrm{GL}=\left(\mathrm{GI}_{\text {mean }} \times \mathrm{C}_{\text {total }}\right)
$$

The rates of glucose appearance were calculated from plasma [6,6- $\left.{ }^{2} \mathrm{H}_{2}\right]$ glucose enrichment (RaT; $\mathrm{T}$ for total glucose) and from plasma ${ }^{13} \mathrm{C}$ glucose enrichment (RaE; E for exogenous glucose) using Steele's equation for non-steady state ${ }^{(27,28)}$ as previously described ${ }^{(22)}$. Endogenous glucose production (EGP) was calculated as RaT-RaE.

Postprandial data were also assessed as area under the curve (AUC) calculated using the trapezoidal method and integrated throughout the experiment $(0-480 \mathrm{~min})$ and between $0-$ $270 \mathrm{~min}$ and $270-480 \mathrm{~min}$. Incremental AUC (iAUC) were calculated using GraphPad Prism (version 4.03; GraphPad Software, San Diego, CA, USA). Glucose peaks were calculated as the maximum glucose concentration following breakfast or lunch ingestion for each subject (consequently it does not correspond to the same time point for each subject).

\section{Statistical analysis}

The results are expressed as means with their standard errors. For each parameter, normality was checked before testing. Statistical significance was inferred at $P<0 \cdot 05$. Differences between groups at baseline and at day 1 were assessed using the Student's unpaired $t$ test.

For postprandial responses, a two-way ANOVA, followed by Bonferroni adjustment was used to compare postprandial peaks and nadir values, as well as AUC, between the groups from day 1 to day 36 and evaluate the main effect of group (LGI compared with HGI), the main effect of the time 
(before dietary intervention compared with after dietary intervention) and the group $\times$ time interaction.

When there was a significant group $\times$ time interaction, the difference within group between day 1 and day 36 was analysed using the Student's paired $t$ test. When GI, GL and change in body weight over the 5 weeks were used as covariates, same statistical results were obtained for the comparison of metabolic parameters from day 1 to day 36 between the groups. Correlation between variables was studied using the $Z$-test.

All statistical analyses were performed using Statview v 5.0 (SAS Institute, Cary, NC, USA) software.

\section{Results}

Dietary survey data, mean glycaemic index and mean glycaemic load

These results have been described previously ${ }^{(18)}$. In summary, the diets were well accepted and tolerated by the subjects, and the dietary surveys indicated good compliance of subjects in both the groups. In comparison with the HGI group, the LGI group presented a trend to increased satiety before lunch, but this was not significant.

While there were no significant differences in GI and GL between groups at baseline, after a 5-week nutritional intervention, the LGI group reached the defined LGI target $(46.5$ (SEM 0.3) for $<50)$ with a significant decrease in mean GI $(P=0 \cdot 001)$. In the HGI group, the defined HGI target (66.3 (SEM 0.6) for > 70) was not reached; GI remained high and was not significantly different to baseline value. The difference in mean GI between the LGI and HGI groups was significant after 5 weeks of diet $(P=0.0001)$. There was no significant variation in energy intake, protein, fat and carbohydrate distributions in both the groups during the trial or between groups at baseline and after 5 weeks of nutritional intervention. There was no difference in dietary fibre intake between groups at baseline. However, the dietary surveys did show a significant increase in dietary fibre intake in the LGI group $(P=0.0001)$, while no significant difference was noticed in the HGI group. No subject was reported as underreporting when using Goldberg's cut-off limits ${ }^{(29)}$.

Comparison of the acute postprandial responses to a low glycaemic index or high glycaemic index breakfast and a standardised lunch

Metabolites and hormones. There was a significant difference in glycaemic response between the HGI and the LGI groups over the $270 \mathrm{~min}$ after ingestion of the two kinds of breakfasts (Fig. 1(a)). In fact, glucose peaks and glycaemia curves between T30 and T180 min were significantly lower in the LGI group than in the HGI group (glucose peak: 7.06 (SEM 0.29$) \mathrm{mmol} / \mathrm{l}$ in the LGI group $v$. 7.85 (SEM $0.17) \mathrm{mmol} / \mathrm{l}$ in the HGI group). Consequently, the glucose AUC and the glucose iAUC following LGI breakfast were significantly lower (8 and $33 \%$, respectively, $P=0.01)$.
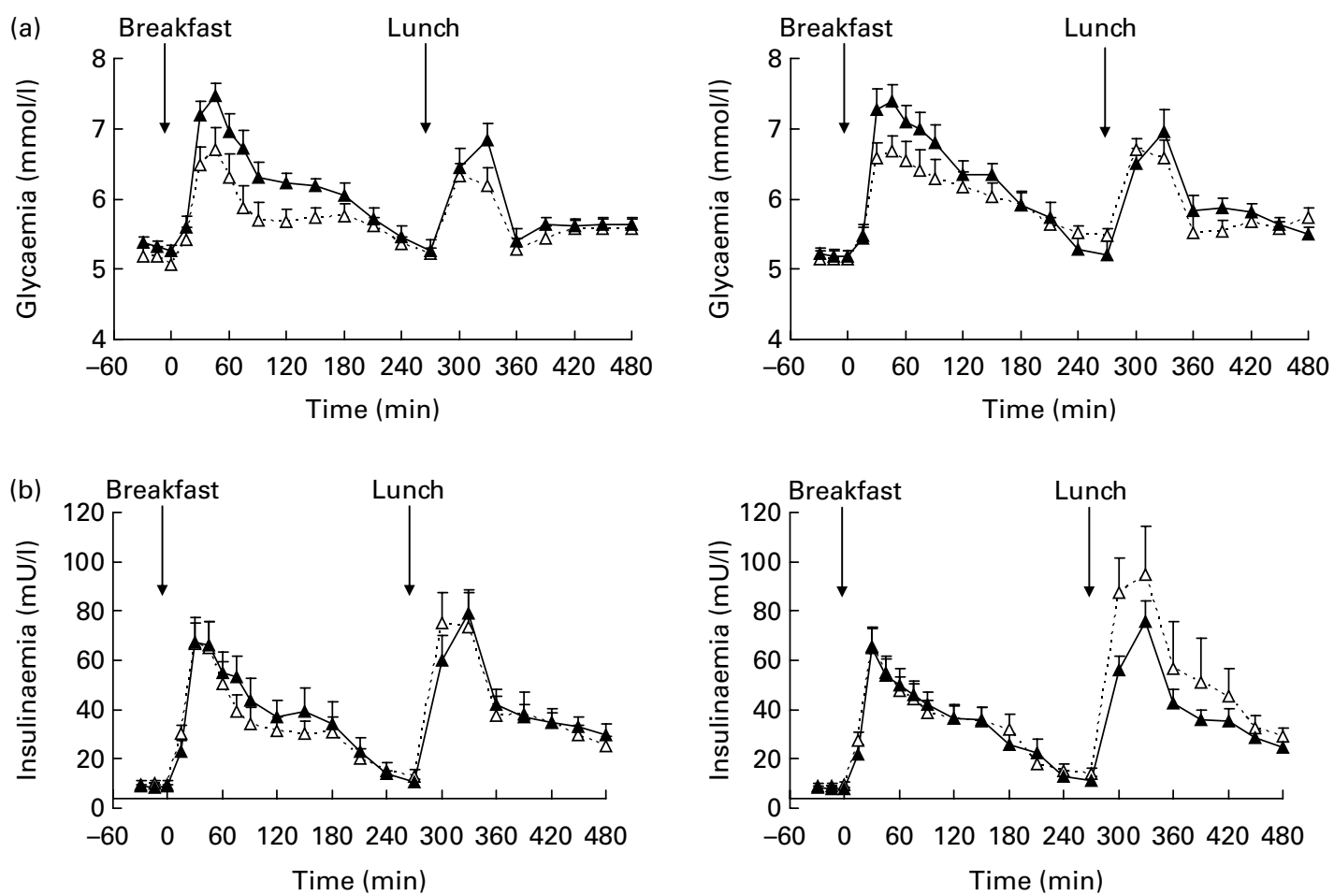

Fig. 1. Means with their standard errors plasma glucose concentration and (a) and plasma insulin (b) for 480 min after subjects ingested either a low glycaemic index breakfast $(\triangle, n 19)$ or a high glycaemic index (HGI) breakfast $(\boldsymbol{\Lambda}, n 19)$ at days 1 and 36 following GI intervention. A standardised HGI lunch was ingested at $t=270 \mathrm{~min}$. At day 1, an unpaired $t$ test showed a significant difference between groups for the post-breakfast glucose peak and $0-270$ min glucose area under the curve (AUC; $\left.{ }^{*} P<0.05\right)$ but no difference between groups for post-lunch glycaemic response or insulinaemic response. Using a two-way ANOVA, a significant main effect of group for the post-breakfast glycaemic peak $(P=0.01)$ and a significant interaction group $\times$ time for the post-lunch insulin peak $(P=0.02)$ and insulin AUC $(P=0.05)$ were seen throughout the 5-week intervention. 
Before the standardised HGI lunch, there was no significant difference between the groups in glucose concentration, which had returned to baseline. Following lunch ingestion, there was a trend to reduced glycaemic response in the LGI group when compared with the HGI group, but this was not significant when considering either the glycaemic peak or the glucose AUC or iAUC (using post-breakfast AUC as covariate). The post-lunch glucose AUC was positively correlated to the post-breakfast glucose AUC and to the T270-min glucose concentration just before lunch $(r 0.79, P<0.0001$ and $r 0 \cdot 38, P=0.02$, respectively). There was also a significant positive correlation between the post-breakfast glucose AUC and the postprandial glucose peak at lunch $(r$ 0.51, $P=0.002$; Table 4).

Plasma insulin response tended to be lower after the LGI breakfasts but this was not significant when considering insulin peak and AUC. Insulin concentration was not different between the groups at $\mathrm{T} 270 \mathrm{~min}$ and insulinaemic response to the lunch was similar for both the groups. Concerning plasma C-peptide concentration, response to the LGI breakfast was lower than that of the HGI breakfast, but the difference was not significant (data not shown).

There was no difference between the groups in lipid profile in response to breakfast and subsequent lunch. Plasma NEFA as well as TAG concentrations during the trial (data not shown) were not altered by the type of breakfast ingested. At T270 min, just before lunch ingestion, there were no significant difference between NEFA concentrations in either group; these parameters had not returned to baseline values at $\mathrm{T} 480 \mathrm{~min}$.

Glucose turnover. Fig. 2 shows the changes in RaE (Rate of appearance of Exogenous glucose), RaT (Rate of appearance of Total glucose) during the 270-min test after the ingestion of the HGI or of the LGI breakfast. RaE kinetics was dramatically altered by the kind of breakfast ingested, as seen in Fig. 2(a). After the HGI breakfast, the rate of exogenous glucose appearance reached a peak at T30 min (4.08 (SEM $0.25) \mathrm{mg} / \mathrm{kg}$ per $\mathrm{min}$ ) and then decreased slightly. After the LGI breakfast, the RaE increased to $\mathrm{T} 45 \mathrm{~min}$ and then remained steady $(<2.5 \mathrm{mg} / \mathrm{kg}$ per $\mathrm{min})$. The overall rate of exogenous glucose appearance integrated over $270 \mathrm{~min}$ (AUC) was also significantly different between the groups: 58.5 (SEM 3.2) g/270 min of exogenous glucose appeared in plasma after the HGI breakfast $v .38 .3$ (SEM 1.9) g/270 min after the LGI breakfast $(P<0.0001)$. The kinetics of disappearance of exogenous glucose was parallel to the $\mathrm{RaE}$ kinetics, and there was also a significant difference in the rate of disappearance of exogenous glucose AUC between the groups $(P<0 \cdot 0001$, data not shown).
The RaT increased in parallel in the two groups following breakfast ingestion with a peak at T30 min. Then RaT decreased until T270 min in both the groups, but remained steadier in the LGI group. After integration of the area under the RaT curve, we calculated the estimated quantity of total glucose which appeared in plasma over the $270 \mathrm{~min}$ following breakfast ingestion. The quantity of total glucose appearing in plasma following LGI breakfast was significantly lower than that following HGI breakfast (RaT AUC: 62.2 (SEM 2) $\mathrm{g} / 270 \mathrm{~min}$ in the LGI group $v .68 .4$ (SEM 2) g/ $270 \mathrm{~min}$ in the HGI group, $P=0.03$ ).

Similarly, the overall rate of disappearance of total glucose was significantly lower in the LGI group $(P=0.03$; data not shown) and the RdT kinetics remained parallel to RaT kinetics in all the groups (data not shown).

EGP was calculated by subtracting RaE from RaT at each time point. As a consequence, EGP appeared to be significantly less inhibited after the ingestion of the LGI breakfast $(P<0.003)$. Over the $270 \mathrm{~min}$ of the test, the quantity of EGP was 24.8 (SEM 1.8) g/270 min for the LGI breakfast, whereas it was 16.4 (SEM 1.9) $\mathrm{g} / 270 \mathrm{~min}$ for the HGI one (data not shown).

Substrate oxidation. There was significant lower total carbohydrate oxidation after the LGI breakfast (39 (SEM 2) in the LGI group v. 45 (SEM 3) in the HGI group). But no significant differences were found between breakfasts with regard to total lipid oxidation. There were no differences between the groups in total carbohydrate and lipid oxidation after lunch ingestion (270-480 min; data not shown).

\section{Effect of a 5-week low glycaemic index or high glycaemic index diet on postprandial responses to a low glycaemic index or high glycaemic index breakfast and a standardised lunch} Metabolites and hormones. When considering glucose
response to breakfast, there was no significant group $\times$ time
interaction for the post-breakfast glucose AUC and glucose
iAUC, no main effect of time or of group for the glucose
AUC. There was a significant main effect of group and of
time for the glucose iAUC (between day 1 and day
$36,+31 \%$ in the LGI group and $+15 \%$ in the HGI group,
$P=0 \cdot 01$ ). There was a significant effect of group for the
post-breakfast glycaemic peak (glucose peak at day $36: 7 \cdot 37$
(SEM $0 \cdot 21)$ mmol/l in the LGI group $v$. $7 \cdot 92$ (SEM
$0 \cdot 28)$ mmol/l in the HGI group, $P=0 \cdot 01$ ). At day 36 , post-
breakfast glucose AUC and glucose iAUC were lower in the
LGI group compared with the HGI group but this was not sig-
nificant $(P=0 \cdot 4$ and $0 \cdot 06$, respectively). Following lunch
ingestion, there was no main effect of group and no significant
group $\times$ time interaction for glucose response, but there was a

Table 4. Correlation in the whole study group between post-breakfast glucose response and post-lunch glucose response at days 1 and 36

\begin{tabular}{lcccccc}
\hline & \multicolumn{2}{c}{ Day 1} & & \multicolumn{2}{c}{ Day 36 } \\
\cline { 3 - 3 } \cline { 5 - 6 } & & $r$ & $p$ & & $R$ & $p$ \\
\hline AUC glycaemia post-lunch $\times$ AUC glycaemia post-breakfast & & 0.79 & $<0.0001$ & & 0.47 & 0.004 \\
AUC glycaemia post-lunch $\times$ glycaemia T270 & & 0.38 & 0.02 & & 0.19 & 0.24 \\
Glucose peak at lunch $\times$ AUC glycaemia post-breakfast & & 0.51 & 0.002 & & 0.33 & 0.04 \\
\hline
\end{tabular}

$r$, correlation coefficient; AUC, area under the curve. 
(a)

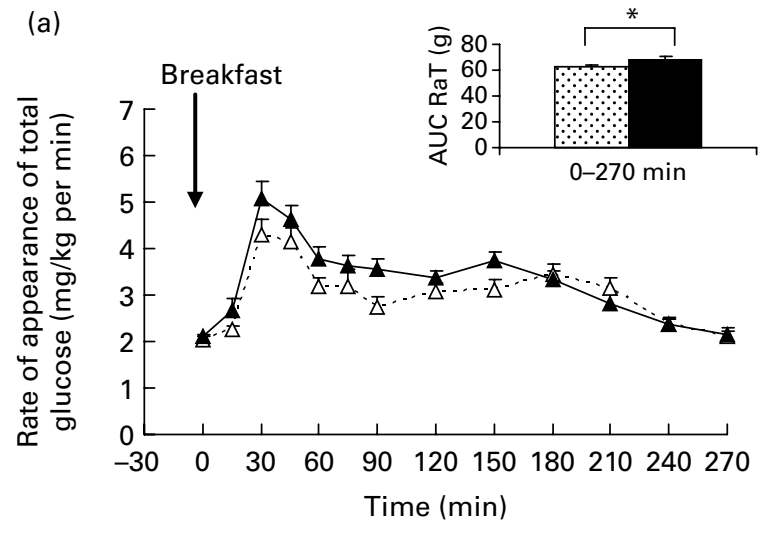

(b)

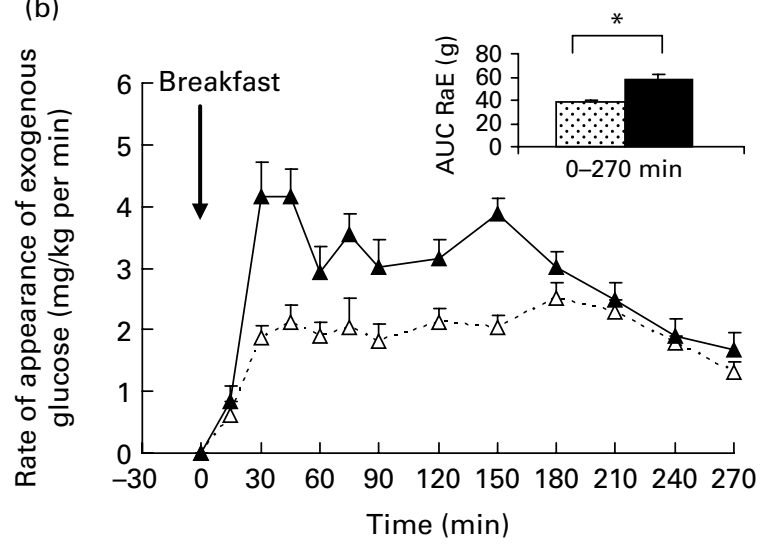

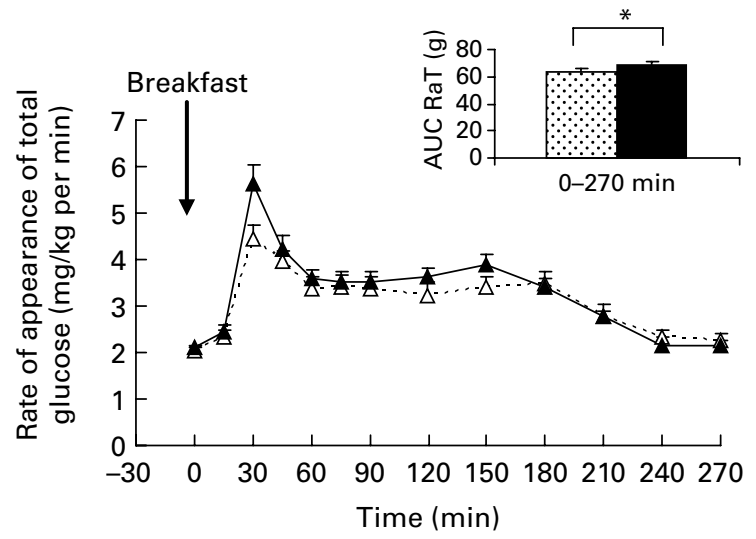

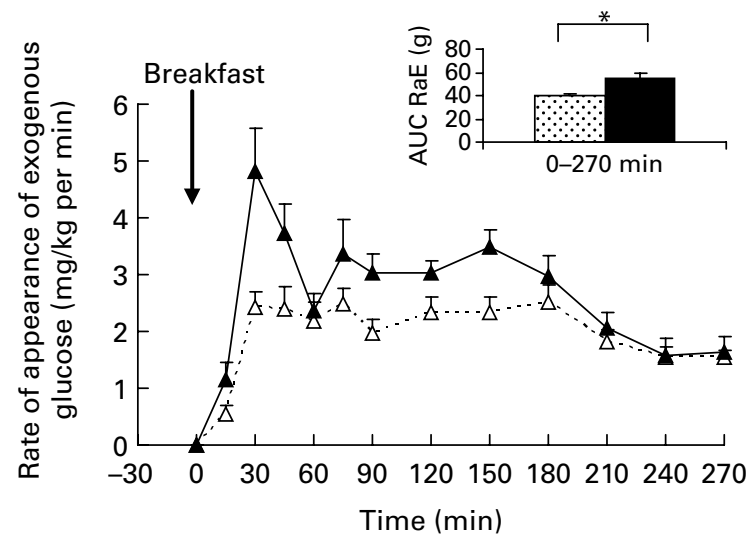

Fig. 2. Means with their standard errors rate of appearance and 270-min plasma appearance (area under the curve, AUC) of total glucose (RaT, (a)), of exogenous glucose $(\mathrm{RaE},(\mathrm{b}))$ after subjects ingested either a low glycaemic index breakfast $(\Delta, n$ 19) or a high glycaemic index (HGI) breakfast $(\boldsymbol{\Lambda}, n$ 19) at days 1 and 36 following Gl intervention. At day 1, an unpaired $t$ test showed a significant difference between groups for the post-breakfast rate of appearance of exogenous glucose (RaE) area under the curve (AUC) and rate of total glucose appearance (RaT) AUC ( $P<0.05)$. Using a two-way ANOVA, a significant main effect of group for the RaE AUC, RaT AUC and endogenous glucose production AUC was seen throughout the 5-week intervention $(P<0.0001,0.0001, P=0.01$, respectively).

significant time effect for the post-lunch glucose peak and for the post-lunch glucose AUC and iAUC $(P=0.04,0.01$ and $P<0 \cdot 0001$, respectively). On day 36 , post-breakfast glucose AUC was positively correlated to post-lunch glucose AUC ( $r 0.47, P=0.004$ ), as well as to the postprandial glucose peak at lunch $(r 0.33, P=0.04)$.

With respect to insulin response to breakfast, there was no significant group $\times$ period interaction and no main effect of group or time, either for the insulin peak or the post-breakfast insulin AUC. After lunch ingestion, there was a significant group $\times$ period interaction for the insulin peak $(P=0.02)$ and for the post-lunch insulin AUC $(P=0 \cdot 04)$. For the LGI group, post-lunch insulin peak and post-lunch insulin AUC were significantly higher at day 36 when compared with day $1(P=0.04$ and $0 \cdot 01$, respectively). But there was no difference in post-lunch insulin peak or post-lunch insulin AUC between the groups at day 36 .

No group $\times$ time interaction and no main effect of group or time were found for $\mathrm{C}$ peptide, NEFA and TAG concentrations (baseline concentrations and postprandial responses, data not shown).

Glucose turnover. No significant group $\times$ time interaction and no main effect of time were found for the $\mathrm{RaE}$ and rate of disappearance of exogenous glucose, but a significant main effect of group appeared over the 5-week intervention dietary trial $(P<0 \cdot 0001)$. At day 36 , the $\mathrm{RaE}$ and rate of disappearance of exogenous glucose curves and associated AUC were still significantly lower in the LGI group $(P=0.0004)$. In the same way, there were no significant group $\times$ time interaction for the RaT and RdT and no main effect of time, but there was a significant main effect of group $(P=0.06$ and 0.05 , respectively). The RaT and RdT curve remained lower in the LGI group at days 1 and 36, but the difference between the groups at day 36 was no longer significant $(P=0 \cdot 1)$. As for EGP, no significant group $\times$ time interaction and no main effect of time was found, but a significant main effect of group $(P=0 \cdot 01)$ remained. Following the 5-week dietary intervention trial, EGP in the LGI group tended to remain less inhibited at day 36 , but this was no longer significant ( $P=0.07$; data not shown).

Substrate oxidation (data not shown). There was no main effect of group or of time and no significant group $\times$ period interaction, when considering carbohydrate or lipid oxidation (baseline and following breakfast and lunch). Carbohydrate oxidation decreased in the two groups after the 5-week diet, but the differences between day 1 and day 36 in each group 
were not significant. In parallel, lipid oxidation was significantly increased in the HGI group between day 1 and day $36(P=0.04)$; the increase in the LGI group was not significant.

\section{Discussion}

In the present study in healthy subjects, a LGI breakfast rich in SAG decreased acute postprandial glucose availability and metabolic response compared with a HGI breakfast. The postprandial glucose response to the breakfast appeared to determine glucose response to the subsequent lunch. This effect of the LGI breakfast on glycaemic response was not accentuated by the 5-week LGI intervention diet.

The cereal products ingested for breakfast did not differ in macronutrient distribution and quantity but differed in term of SAG composition and thus in term of GI as previously shown ${ }^{(16,30,31)}$. These properties are correlated to in vitro starch digestibility and are dependent of processing ${ }^{(32)}$. This way, the observed effect could not be attributed to a modification in protein or lipid content. The present study, thanks to the monitoring of glucose kinetics, provides evidence that the SAG content of the LGI breakfast induced a significant decrease in exogenous glucose appearance, which contributed to the LGI effect. In parallel, EGP was less inhibited and balanced the total glucose response. Indeed, differences in EGP, due to the physiological glucose regulation in healthy subjects, lowered the final impact on glycaemia. This compensatory effect may not be observed in diabetic subjects, and this could explain the improvement in glucose control with low GI diets in diabetic subjects or in subjects with poor glycaemic control. In the present parallel study, the subjects consume either the LGI or the HGI breakfast. Thus, to adjust for inter-individual differences, iAUC was calculated and the same statistical results were obtained. Previous studies using glucose-stable isotope analysis have tried to explain the moderate glycaemic response associated to low GI products in term of plasma glucose appearance and disappearance. Comparing the ingestion of breakfasts with different GI, Schenk et al. ${ }^{(15)}$ showed that the different GI of breakfast cereals could be partially related to the different rates of glucose removal from blood by tissue as a result of stimulation of insulin secretion and not to the difference in glucose appearance in plasma. But in another study, in which exogenous and endogenous glucose kinetics were measured, the addition of $\beta$-glucan to a polenta meal did prolong insulin secretion and reduced glycaemic response and the rate of appearance of exogenous and total glucose, with no significant alteration in the rate of glucose disposal ${ }^{(33)}$. In the present study, the ingestion of the LGI breakfast induced a decrease in both the appearance and removal of exogenous and total glucose, suggesting that different GI could be related to both metabolic mechanisms.

In the meta-analysis conducted by Livesey et al. ${ }^{(5)}$, the available carbohydrate content was shown to have an impact on glycaemic control, even if it was weaker than GI or GL. In the present study, the lower glycaemic response at lunch associated to the lower glycaemic response at breakfast is consistent with results from previous studies ${ }^{(6-8,14,34)}$. A study comparing the effect of the GI and indigestible carbohydrateresistant starch and dietary fibre content of cereal-based breakfasts on day-long glucose tolerance at a second meal (lunch) concluded that the content of fermentable carbohydrates per se did not influence second-meal glucose tolerance ${ }^{(7)}$. This beneficial effect has been allocated for some part of the ability of certain carbohydrates to produce slow and sustained glycaemia. This could be compared to the present study in which the reduced glycaemic response was associated with the decreased appearance of exogenous glucose. The significant positive correlation found between glucose response to the breakfast (T0-270 min AUC) and glucose response to the standardised lunch (T270-480 min AUC) showed that the kind of breakfast ingested can impact on the glucose tolerance in the short term. This is in accordance with results from a previous study by Nilsson et al. ${ }^{(34)}$. The authors tested glucose tolerance and response to breakfasts differing in GI and indigestible carbohydrate and found a positive correlation between the glucose response to breakfast and the glucose response to lunch, as in the present results, but a negative correlation between glucose before the start of the lunch and the glucose response to lunch. They concluded on the major input of GI on this second-meal effect, with an independent effect of colonic fermentation. The GI was in fact a major determinant of second-meal effect in both Nilsson's and our own study, but we did not find the same impact of glucose concentration just before the second meal.

In the present study, the metabolic effect of LGI $v$. HGI diet was investigated over 5 weeks. Interestingly, after the 5-week dietary intervention, a significant decrease in GI was obtained in the LGI group, by replacing usual starchy products in diet by LGI products. This showed that it is possible to implement such a dietary intervention based on simple dietary advice and a few LGI products. These modifications in the mean GI of diet in the two groups did not affect the intake of other nutrients, either in quantity or proportion. It should be noted that in the LGI group there was also a trend to a decrease in hunger sensation between meals ${ }^{(18)}$. This effect may be due to the consumption of food richer in dietary fibre and thus more satiating in this group $^{(35)}$. The mean GL of food in the LGI group decreased in parallel to the mean GI and it is difficult to distinguish the effects due to GI and/or GL, as already underlined in other studies ${ }^{(5)}$. Following the 5-week dietary intervention, no significant effect was noticed on baseline glucose and insulin concentrations. This is consistent with conclusions of Livesey's ${ }^{(5)}$ meta-analysis, in which the authors concluded that LGI products could have an effect on baseline glucose tolerance parameters in subjects with baseline glycaemia $>5 \mathrm{~mm}$ or with poor glucose control. Concerning the post-breakfast glycaemic response, the difference between the groups (LGI $v$. HGI) was maintained over the 5 weeks (no significant group $\times$ time interaction) but tended to decrease over time. Indeed, at day 36, post-breakfast AUC and iAUC were lower in the LGI group but this was not significant. The two cereal products still differed significantly in term of exogenous glucose appearance in plasma, but the endogenous production was not significantly different between the groups. However, at day 36, the glycaemic response to lunch remained correlated to the glycaemic response to breakfast. But in the LGI group, insulin response to the lunch was significantly higher. Thus, the lowest glycaemic response at lunch associated to the lowest glycaemic response at breakfast (potential secondmeal effect) was maintained over weeks but this seems to be at the expense of the insulin profile. One hypothesis could be that 
the LGI group may present an improvement in $\beta$-cell function, as it has been demonstrated by Wolever et al. ${ }^{(13)}$. But, when assessed by homeostasis model assessment $\beta$-cell function, no difference was found in $\beta$-cell function between the groups and over time (data not shown). Several papers have reported long-term beneficial effects of low-GI foods on glucose metabolism and insulin sensitivity in type 2 diabetic subjects and healthy subjects ${ }^{(5,36-38)}$. Moreover, the reduced glycaemic response induced by LGI products could present a potential beneficial effect on oxidative stress with a reduction of glucose excursion, as already shown ${ }^{(39)}$. In the present study, the 5-week dietary intervention may have equalised glycaemic profiles by regularising breakfast intake in both the groups and thus tended to balance the difference between LGI and HGI metabolic effects.

LGI foods were thought to potentially promote fat oxidation compared to carbohydrate oxidation, through their action on insulin response ${ }^{(40,41)}$. But the present results did not show any major change of nutrient oxidation and of respiratory exchange rate following GI modification. Reviewing the GI effects on nutrient oxidation (short-, mid- and long-term), Diaz et al. came up with the statement that fuel partitioning was not affected by different glycaemic features ${ }^{(42)}$. The metabolic changes induced by a LGI intervention seemed insufficient to initiate a significant effect on lipid oxidation.

In conclusion, modulation of postprandial glucose availability at breakfast is shown to be an important factor in daylong metabolic control in healthy subjects, as it decreases plasma exogenous glucose appearance and improves glucose control at subsequent lunch. After 5 weeks, such acute effects on glucose metabolism were maintained but remain to be confirmed in the longer term.

\section{Acknowledgements}

J.-A. N, A. d. R and S. N. were responsible for the study design and coordination, collection and analysis of data, statistical analysis, writing and evaluation of the paper. M. S. was responsible for the diet design, dietary surveys and analysis. S. V. provided expert analysis and knowledge of cereal products. M. D., V. S. and S. N. provided technical expertise in MS analysis of glucose tracers. M. L. was responsible for study coordination, study design and supervised the writing of the paper. We thank the staff of the Centre de Recherche en Nutrition Humaine Rhône-Alpes and more particularly Christine Maitrepierre, Jocelyne Peyrat, Corinne LouchePélissier for their excellent technical assistance in subject recruitment, sample collection and analysis. Breakfast cereals and biscuits were supplied by Danone Vitapole, France. The present study was part of the EUROSTARCH project, contract no. QLK1-2001-00431, supported by the European Union. S. V. was an employee of Danone Vitapole. The other authors have no conflict of interest to declare.

\section{References}

1. Coutinho M, Gerstein HC, Wang Y, et al. (1999) The relationship between glucose and incident cardiovascular events. A metaregression analysis of published data from 20 studies of 95,783 individuals followed for 12.4 years. Diabetes Care 22, $233-240$.
2. Jenkins DJ, Kendall CW, Augustin LS, et al. (2002) Glycemic index: overview of implications in health and disease. Am J Clin Nutr 76, 266S-273S.

3. Salmeron J, Manson JE, Stampfer MJ, et al. (1997) Dietary fiber, glycemic load, and risk of non-insulin-dependent diabetes mellitus in women. JAMA 277, 472-477.

4. Augustin LS, Franceschi S, Jenkins DJ, et al. (2002) Glycemic index in chronic disease: a review. Eur J Clin Nutr 56, 1049-1071.

5. Livesey G, Taylor R, Hulshof $\mathrm{T}$, et al. (2008) Glycemic response and health - a systematic review and meta-analysis: relations between dietary glycemic properties and health outcomes. Am J Clin Nutr 87, 258S-268S.

6. Wolever TM, Jenkins DJ, Ocana AM, et al. (1988) Second-meal effect: low-glycemic-index foods eaten at dinner improve subsequent breakfast glycemic response. Am J Clin Nutr 48, $1041-1047$.

7. Liljeberg HG, Akerberg AK \& Bjorck IM (1999) Effect of the glycemic index and content of indigestible carbohydrates of cereal-based breakfast meals on glucose tolerance at lunch in healthy subjects. Am J Clin Nutr 69, 647-655.

8. Liljeberg H \& Bjorck I (2000) Effects of a low-glycaemic index spaghetti meal on glucose tolerance and lipaemia at a subsequent meal in healthy subjects. Eur J Clin Nutr 54, 24-28.

9. Nilsson A, Granfeldt Y, Ostman E, et al. (2006) Effects of GI and content of indigestible carbohydrates of cereal-based evening meals on glucose tolerance at a subsequent standardised breakfast. Eur J Clin Nutr 60, 1092-1099.

10. Brighenti F, Benini L, Del Rio D, et al. (2006) Colonic fermentation of indigestible carbohydrates contributes to the secondmeal effect. Am J Clin Nutr 83, 817-822.

11. Miles JM (2008) A role for the glycemic index in preventing or treating diabetes? Am J Clin Nutr 87, 1-2.

12. Brand-Miller JC, Petocz P \& Colagiuri S (2003) Meta-analysis of low-glycemic index diets in the management of diabetes: response to Franz. Diabetes care 26, 3363-3364.

13. Wolever TM, Mehling C, Chiasson JL, et al. (2008) Low glycaemic index diet and disposition index in type 2 diabetes (the Canadian trial of carbohydrates in diabetes): a randomised controlled trial. Diabetologia 51, 1607-1615.

14. Jenkins DJ, Wolever TM, Taylor RH, et al. (1982) Slow release dietary carbohydrate improves second meal tolerance. Am J Clin Nutr 35, 1339-1346.

15. Schenk S, Davidson CJ, Zderic TW, et al. (2003) Different glycemic indexes of breakfast cereals are not due to glucose entry into blood but to glucose removal by tissue. Am J Clin Nutr 78, $742-748$

16. Englyst KN, Englyst HN, Hudson GJ, et al. (1999) Rapidly available glucose in foods: an in vitro measurement that reflects the glycemic response. Am J Clin Nutr 69, 448-454.

17. McMillan-Price J, Petocz P, Atkinson F, et al. (2006) Comparison of 4 diets of varying glycemic load on weight loss and cardiovascular risk reduction in overweight and obese young adults: a randomized controlled trial. Arch Intern Med 166, 1466-1475.

18. de Rougemont A, Normand S, Nazare JA, et al. (2007) Beneficial effects of a 5-week low-glycaemic index regimen on weight control and cardiovascular risk factors in overweight non-diabetic subjects. Br J Nutr 98, 1288-1298.

19. Foster-Powell K, Holt SH \& Brand-Miller JC (2002) International table of glycemic index and glycemic load values: 2002. Am J Clin Nutr 76, 5-56.

20. Arock M, Desnault H, Viars P, et al. (1985) Determination of total nitrogen in biological milieux by chemoluminescence: a comparison with the reference method. Ann Biol Clin (Paris) 43, 872-874.

21. Ferrannini E (1988) The theoretical bases of indirect calorimetry: a review. Metabolism 37, 287-301. 
22. Tissot S, Normand S, Guilluy R, et al. (1990) Use of a new gas chromatograph isotope ratio mass spectrometer to trace exogenous ${ }^{13} \mathrm{C}$ labelled glucose at a very low level of enrichment in man. Diabetologia 33, 449-456.

23. Bier DM, Arnold KJ, Sherman WR, et al. (1977) In vivo measurement of glucose and alanine metabolism with stable isotopic tracers. Diabetes 26, 1005-1015.

24. Dejongh DC \& Hanessian S (1965) Characterization of amino sugars by mass spectrometry. J Am Chem Soc 87, 3744-3751.

25. Thivend P MC \& Guilbot A (1972) Determination of starch with glucoamylase. In Methods in Carbohydrate Chemistry, pp. 100-105 [RL Whisler, editor]. New York/London: Academic Press.

26. Normand S, Pachiaudi C, Khalfallah Y, et al. (1992) ${ }^{13} \mathrm{C}$ appearance in plasma glucose and breath $\mathrm{CO}_{2}$ during feeding with naturally ${ }^{13} \mathrm{C}$-enriched starchy food in normal humans. Am J Clin Nutr 55, 430-435.

27. De Bodo RC, Steele R, Altszuler N, et al. (1963) On the hormonal regulation of carbohydrate metabolism: studies with ${ }^{14} \mathrm{C}$ glucose. Recent Prog Horm Res 19, 45-48.

28. Proietto J, Rohner-Jeanrenaud F, Ionescu E, et al. (1987) Nonsteady-state measurement of glucose turnover in rats by using a one-compartment model. Am J Physiol 252, E77-E84.

29. Goldberg GR, Black AE, Jebb SA, et al. (1991) Critical evaluation of energy intake data using fundamental principles of energy physiology: 1 . Derivation of cut-off limits to identify under-recording. Eur J Clin Nutr 45, 569-581.

30. Englyst KN, Hudson GJ \& Englyst HN (2000) Starch analysis in food. In Encyclopedia of Analytical Chemistry, pp. 4246-4262 [R Meyers, editor]. Chichester: John Wiley \& Sons Ltd.

31. Seal CJ, Daly ME, Thomas LC, et al. (2003) Postprandial carbohydrate metabolism in healthy subjects and those with type 2 diabetes fed starches with slow and rapid hydrolysis rates determined in vitro. Br J Nutr 90, 853-864.
32. Garsetti M, Vinoy S, Lang V, et al. (2005) The glycemic and insulinemic index of plain sweet biscuits: relationships to in vitro starch digestibility. J Am Coll Nutr 24, 441-447.

33. Nazare J-A, Normand S, Triantafyllou AO, et al. (2007) Modulation of the postprandial phase by beta-glucan in overweight subjects: effects on glucose and insulin kinetics. Mol Nutr Food Res 53, 361-369.

34. Nilsson AC, Ostman EM, Granfeldt Y, et al. (2008) Effect of cereal test breakfasts differing in glycemic index and content of indigestible carbohydrates on daylong glucose tolerance in healthy subjects. Am J Clin Nutr 87, 645-654.

35. Howarth NC, Saltzman E \& Roberts SB (2001) Dietary fiber and weight regulation. Nutr Rev 59, 129-139.

36. Jenkins DJ, Wolever TM, Buckley G, et al. (1988) Lowglycemic-index starchy foods in the diabetic diet. Am J Clin Nutr 48, 248-254.

37. Brand JC, Colagiuri S, Crossman S, et al. (1991) Low-glycemic index foods improve long-term glycemic control in NIDDM. Diabetes Care 14, 95-101.

38. Wolever TM, Jenkins DJ, Vuksan V, et al. (1992) Beneficial effect of low-glycemic index diet in overweight NIDDM subjects. Diabetes Care 15, 562-564.

39. Food and Drug Administration (2006) Food labeling: health claims; soluble dietary fiber from certain foods and coronary heart disease. Final rule. Fed Regist 71, 29248-29250.

40. Brand-Miller JC, Holt SH, Pawlak DB, et al. (2002) Glycemic index and obesity. Am J Clin Nutr 76, 281S-285S.

41. Wee SL, Williams C, Tsintzas K, et al. (2005) Ingestion of a high-glycemic index meal increases muscle glycogen storage at rest but augments its utilization during subsequent exercise. J Appl Physiol 99, 707-714.

42. Diaz EO, Galgani JE \& Aguirre CA (2006) Glycaemic index effects on fuel partitioning in humans. Obes Rev 7, 219-226. 